\title{
IMPROVING SAFETY WITH OBSTACLE DETECTION AND TRACK FOLLOWING CAR USING SENSOR, GPS AND GSM
}

\author{
Sangita N.Gujar ${ }^{1}$, Jagruti R.Panchal ${ }^{2}$, Lalita K.Wani ${ }^{3}$ \\ ${ }^{1}$ M.E.Student, E \&TC, Siddhant College of Engineering, Pune, Maharashtra, India \\ ${ }^{2}$ Associate Professor, E \&TC, Siddhant College of Engineering, Pune, Maharashtra, India \\ ${ }^{3}$ Associate Professor, E \&TC, Siddhant College of Engineering, Pune, Maharashtra, India
}

\begin{abstract}
Through this Project our expectation is that the car should start when authorized persons send predefined message on the External SIM. Otherwise the car is locked. Smart vehicle sends current locations on the different predefined mobile numbers. In this situation owner calls on External SIM for the first time, then the car should stop \& when the user calls second time, then the vehicle will start automatically. Also, When the car is in motion and suddenly Obstacle occurring in front of the vehicle, then the sensor should detect barrier, (may be small or big) car should break automatic. And try to search alternate path in case of an accident. For immediate help and send that message to an owner and care center. In that Short Message Service it is should send current place latitude and longitude, Time, Speed in knots, Date, obtained from Global Positioning, Therefore, using that latitude and longitude, we find location on earth and we may provide fast help in an emergency. The Car should also break if the car is on wrong driving track and automatically re-track car. The Micro Controller PIC18F26K22 interfaces with Infrared sensors and Ultrasonic sensor that continuously detect, track, and the vehicle is in motion. If an Infrared sensor output detects off road path, then Micro controller immediately slows down the vehicle speed and try to move the vehicle back on path depending on track sensor output. In this project Micro controller is programmed using embedded C language and built using MPLAP.
\end{abstract}

Keywords: Infrared Sensor, Ultrasonic Sensor, PIC Microcontroller, GSM, GPS

\section{INTRODUCTION}

To improve overall system, Microcontroller featured with GSM and GPS interface.GPS device received valid GPS signals from satellite and send calculated longitude and latitude and speed of the vehicle to Microcontroller at every one second. Microcontroller continuously monitor the speed received from GPS if there is a huge predefined difference between two consecutive readings of speed from the GPS is treated as accident condition. For proper observation, we have designed a lookup table which shows that your initial speed \& speed after one second that means some threshold value are predefined. [1]

In such event Microcontroller will send respective AT commands to GSM modem interfaced to take action for Sending text SMS to the predefined owners mobile number. This text message includes current latitude and longitude received from the GPS receiver. [2] On Dynamic Stability and track detection control makes driving on winding and Sliding road conditions safer, by using sensors to detect any of the wheels is losing path. It has the ability to slow down car speed, helping the car to regain its grip on the path. If in any unexpected situation, the car shows a direction of turning too quickly, then the system automatically slows down the wheel speed to help maintain control. In obstacle detection car will detect any object or other car in its driving path, as any obstacle detects in path it shall automatically reduce the car speed by applying smooth break. Many researchers work in this same area. They developed a different model to minimize the percentage of road accidents. In Traditional system, Microcontroller is used with assembly language, but we have used PIC Microcontroller along with embedded C Language. So that there is no restriction on coding

\subsection{Survey of the Related Research}

In [1], says that if accidental porn is occurring then, GPS sends the location of the accident with some predefined message body of the alert system or to the concern person. PIC18F4550 Microcontroller receives data from the GPS \& encodes that data. That means Microcontroller compare the previous \& current value. Some threshold values are defined as concerns with speed. If the vehicle speed is below the threshold value the we considered as something is happening to the vehicle. The location details sent via GSM module to concern authority on his/her mobile number. We have implemented this paper.

In [2], Authors demonstrates the accident detection \& alert system using GPS \& the GSM system with Microcontroller AT89C52 as a core. He prepares a survey report that shows an 
porn occur because of the various things. The authors decide to nullify the accidental situation because it is difficult to track the accidental situation in the rural area. The whole project assembly is placed inside the vehicle. GPS finds the location of the vehicle. Data shall be sent to MAX232 via RS232 Cable. Vibration \& sound sensors are used efficiently \& the correct way of accident identification.

In [3], have used an IR sensor to detect a front side obstacle of $3 \mathrm{ft}$ and authors also monitors the different toxic gas alert system during in an emergency and send this information in the form of SMS to the authorized person. Gas Sensing, Alarm and GSM Module are interfaced with AT89S52 Microcontroller, through RS232.

In [4], proposed a new approach on to differentiate between human and other obstacle and accidental situation send location.The Passive Infrared sensor is mainly used to detect human or animal .Atmega8 micro-controller is used in this project.

In [5], this system provides a new method of vehicle locking and tracking to identify the theft/ unauthorized person through IR Sensor, GPS and GSM technology. Microcontroller AT89C52 is used as a heart of the system. When an unauthorized person is identified, then owner sends SMS to AT89C52 then all doors and motor engine is locked.

\section{PROPOSED BLOCK DIAGRAM}

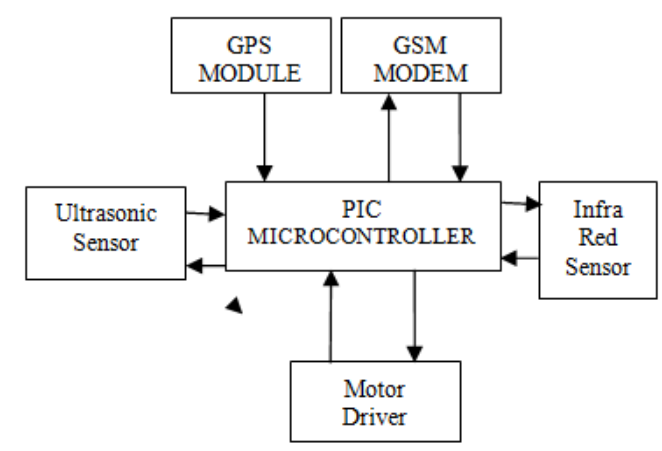

Fig- 1: Block Diagram of Obstacle and Track Detection [12]

The block diagram consists of the Ultrasonic sensor (HCSR04), infrared sensor (LM323), Motor Drive (LM293D), GPS Module (L50) \& GSM Modem (M12). The heart of the system is PIC18F26K22 microcontroller. As soon as any obstacle detected through Ultrasonic sensor microcontroller shall immediately slow down vehicle speed and come to rest till obstacle is in the path. Once the sensor detects nothing is in path controller shall bypass control on breaks. To detect obstacle in vehicle path the sensor is placed in such a way that each cover the maximum area in front of the vehicle chassis and to detect a Human or animal and obstacle either obstacle is small or big. [3][4] When vehicle follows the wrong path, then, Infrared sensors, transmitter \& receiver pairs which are placed at the bottom of the car sense that track and automatically move the car towards the correct path. [6]

\section{EQUIPMENT/PROPOSED BLOCK DIAGRAM}

\subsection{PIC Microcontroller Unit}

The Microcontroller PIC18F26K22 is interfaced with a track sensor that continuously detect, track, the vehicle is in motion if track sensor output detects off road path, then the Microcontroller immediately slow down vehicle speed and try to move the vehicle back on path depending on track sensor output. Also Microcontroller interfaced with Ultrasonic sensors to detect any obstacle is present in vehicle path, is in movement. In addition, to improve overall system,Microcontroller featured with GSM and GPS interface.The PIC18F26K22 is a Low power, High performance, 16 bit Microcontroller with 1024 Bytes Data EEPROM \& nano watt XLP Technology. The PIC18F26K22 provides the following standard features.

- C Compiler optimized architecture/instruction set designed to optimize re-entrant code.

- Self-Programmable under Software Control

- Data EEPROM up to 1024 bytes

- Linear programming memory addressing to 64 Kbytes

- $\quad$ Sleep mode: $20 \mathrm{nA}$, typical

- Linear data memory addressing to 4 Kbytes

- Selectable frequencies, $31 \mathrm{kHz}$ to $16 \mathrm{MHz}$

- In-Circuit Debug (ICD)

- SR Latch:- Multiple Set/Reset input options

- Precision $16 \mathrm{MHz}$ Internal Oscillator Block:- Factory calibrated to $\pm 1 \%$

- $\quad$ Fixed Voltage Reference (FVR) channel

- $\quad$ Peripheral Module Disable

- $\quad$ Fixed Voltage Reference (FVR) with 1.024V,2.048V and $4.096 \mathrm{~V}$ output levels

In addition PIC18F26K22 is designed with $64 \mathrm{MHz}$ performance available using PLL, no external components required, Auto-acquisition capability, Conversion available during Sleep, Independent input multiplexing, Digital-toAnalog Converter (DAC) module, 5-bit rail-to-rail resistive DAC with positive and negative reference selection, Charge Time Measurement Unit (CTMU) module:-Supports capacitive touch sensing for touch screens and capacitive switches, Auto-Shutdown and Auto-Restart, In-Circuit Serial Programming (ICSPTM):- Single-Supply 3V, Extended Watchdog Timer (WDT):- Programmable period from $4 \mathrm{~ms}$ to 131s, Programmable Brown-out Reset (BOR):- With software enable option,- Configurable shutdown in Sleep, and - Nine programmable weak pull-ups 


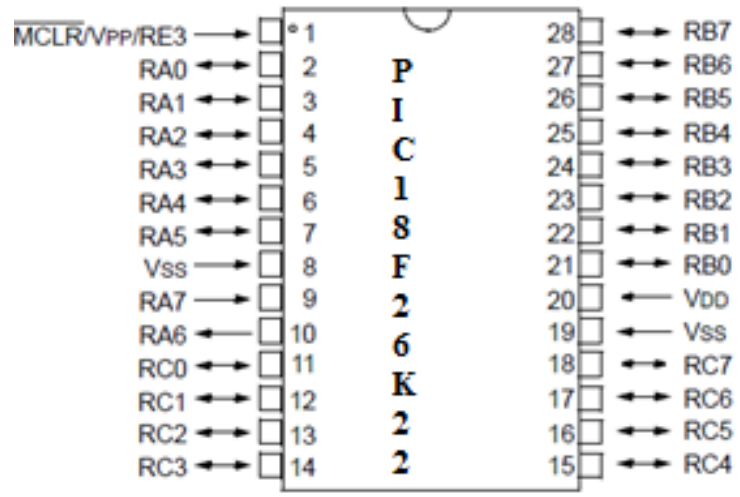

Fig-2:PIC18F26K22 Pin Diagram

\subsection{The Track Sensing}

The demonstration vehicle chassis uses Infra Red (IR) sensors to sense the line tracks; a line-up of 8 Infra Red LEDs (Transmitter) and sensors (Receiver), facing the earth has been used in track detection. The output of the sensors is an analog signal that depends on the amount of light reflected back, this analog signal is given to the comparator to produce Logic $0 \mathrm{~s}$ and Logic 1s that are then fed to the Microcontroller. The Motor Driver has four inputs to control the motion of the vehicle and to enable the inputs which are used to divert the motors on and off.PIC generating Signal of $38 \mathrm{KHz}$ frequency for better determination of the object. Like $600 \mathrm{~mA}$ output current capability per channel, 1.2A Maximum output current per channel, enable facility, over temperature protection, logical " Logic 0" input voltage up to $1.5 \mathrm{v}$ (high noise immunity), internal clamp diodes. We have molten vehicle body as shown in figure 3. So that sensor pairs are placed properly.

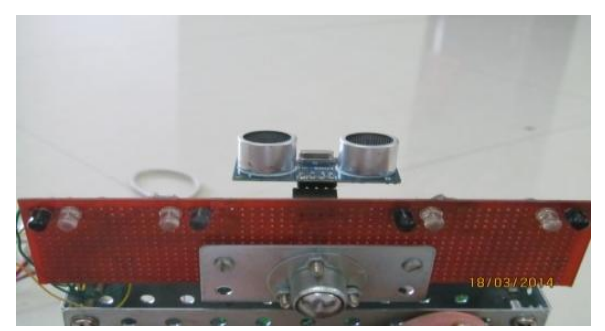

Fig -3: Track Detection setup [12]

IR transmitters are continuously emitting IR rays which may reflect and received by IR receiver. The Output of this IR receiver is connected to the negative input of comparator while the positive input of the comparator is set at a fixed voltage level through variable resistor R12.When the IR receiver detects rays it conducts and the comparator input goes to a low level as effect comparator output goes to high level which is the sense of Microcontroller input. Similarly, all four IR transmitter receiver pair works. IR rays absorbed by Black or similar color and reflects by white or similar color, in firmware logic this change in digital input signal will determine whether IR transmitter on black area or white are this will further decide motor driver actions. LED4, LED5, LED6, LED7 will ON condition when their respective IR receiver detects black portion otherwise LED will OFF if the respective IR receiver detects white portion.

\subsection{Detecting Obstacle}

HC SR-04 Ultrasonic detector module has transmitter and receiver section. This sensor interface with micro controller with one output named as the trigger and one input named as echo. Microcontrollers trigger this sensor at every one second interval and detect for the echo signal on its input pin. If echo signal high width is detected is in predefined range, then obstacle detection is considered and same time motor 1 and motor2 signals to stop running as soon as obstacle removed again motor will start as per IR sensor inputs. [7]

When any obstacles are in range of ultrasonic sensors, the firmware will glow LED3.The Ultrasonic Detector HC-SR04 circuit is a circuit which gives a low output in the absence of Ultrasonic signal when some obstacle come in path Ultrasonic signal reflected back and fall onto the Ultrasonic detector. In such a way that obstacle is detected. Ultrasonic ranging module HC - SR04 provides $2 \mathrm{~cm}-400 \mathrm{~cm}$ non-contact. The measurement function, the ranging accuracy can reach to $3 \mathrm{~mm}$. The modules include ultrasonic transmitters, receiver and control circuit. The basic principle of work:

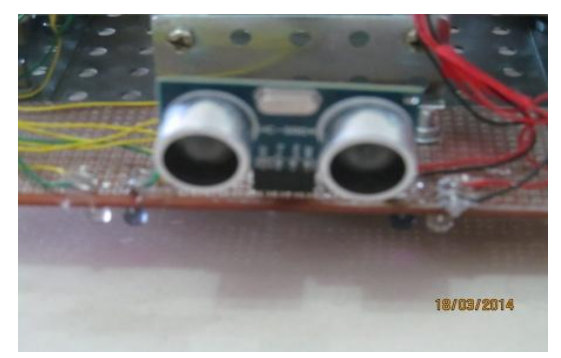

Fig- 4. Detecting Obstacle actual setup [12]

(1) Using IO trigger for at least 10us high level signal,(2) The Module automatically sends eight $40 \mathrm{kHz}$ and detect whether there is a pulse signal back.(3) IF the signal back, through high level , time of high output IO duration is the time from sending ultrasonic to returning.

Test distance $=($ high level time $*$ velocity of sound $(340 \mathrm{M} / \mathrm{S})$ 12 ,

\begin{tabular}{|c|c|c|}
\hline \multirow{2}{*}{ Microcontroller } & Trigger & \multirow{2}{*}{ Ultrasonic sensor } \\
\hline & EChO & \\
\hline
\end{tabular}

Fig-6. Interfacing with Detecting Obstacle 


\subsection{GSM Modem}

The Modem can be controlled by a microcontroller through AT command set. The M12 Quectel GSM Modem used in this system with Dual band 900/1800 MHz Compact "Plug And Play" Dual band GSM modems can be directly connected to the serial port of a Controller through the serial interface. [5] GSM M12 is dual band frequency GSM with UART port which is used for AT command reception. Microcontroller UART1 is connected to the GSM UART on our hardware. Firmware logic flow written such that whenever a Microcontroller detects signal from the ultrasonic sensor as an obstacle detection or sudden change in speed observed from GPS data then Microcontroller will send AT command set on its UART1. This AT commands made for sending SMS to predefined SIM number. E.g. AT+CMGS="98xxxxxxxx" Enter <GPS data>. At the GSM end after receiving this command it will send SMS to SIM number written in command.GSM has SIM card signals of SIM socket connections where we can put the SIM card through which GSM module will register on GSM network.

\begin{tabular}{|c|c|c|c|}
\hline Microcontroller & & $\longrightarrow$ & GSM \\
\hline
\end{tabular}

Fig.6 GSM Interface

GSM provides the following features.

- Dual Band 900 / 1800MHz

- GSM and GPRS Multi slot class -12/10/8

- Dual band GSM/GPRS module with size of $29 \mathrm{~mm} * 29 \mathrm{~mm} * 3.6 \mathrm{~mm}$

- Voice / SMS and Data

- Accepts Standard SIM Card

- Can Be Used On Standard GSM Network Serial Interface

- Operating temperature $-40{ }^{\circ} \mathrm{C}$ to $+80{ }^{\circ} \mathrm{C}$

- Coding scheme - CS 1,2,3,4

- External SIM -3V/1.8V

- One user programmable input/output Port

- GSM 07.05 and 07.07 Other enhanced AT Commands

\subsection{GPS Module}

The GPS module can receive the data by connecting to PIC Microcontroller Universal asynchronous receiver/transmitter (UART1) through serial fashion. L50 GPS module is connected to microcontroller UART2 through the GPS UART port. GPS module is a combo device, with inbuilt antenna. . This location string is the universal format called NEMA protocol. E.g. \$GPRMC,122825.000,A,1828.9146,N ,07353.8977,E,0.04,339.41,110211,,A*60.This string contains latitude, longitude, Date, time and speed. Microcontroller received this data at every one second. On the basis of speed the firmware logic will take the decision to send SMS AT commands on UART1. If speed suddenly changes above 0.90 it will send SMS commands to GSM. When GPS received a valid signal firmware will glow LED2.

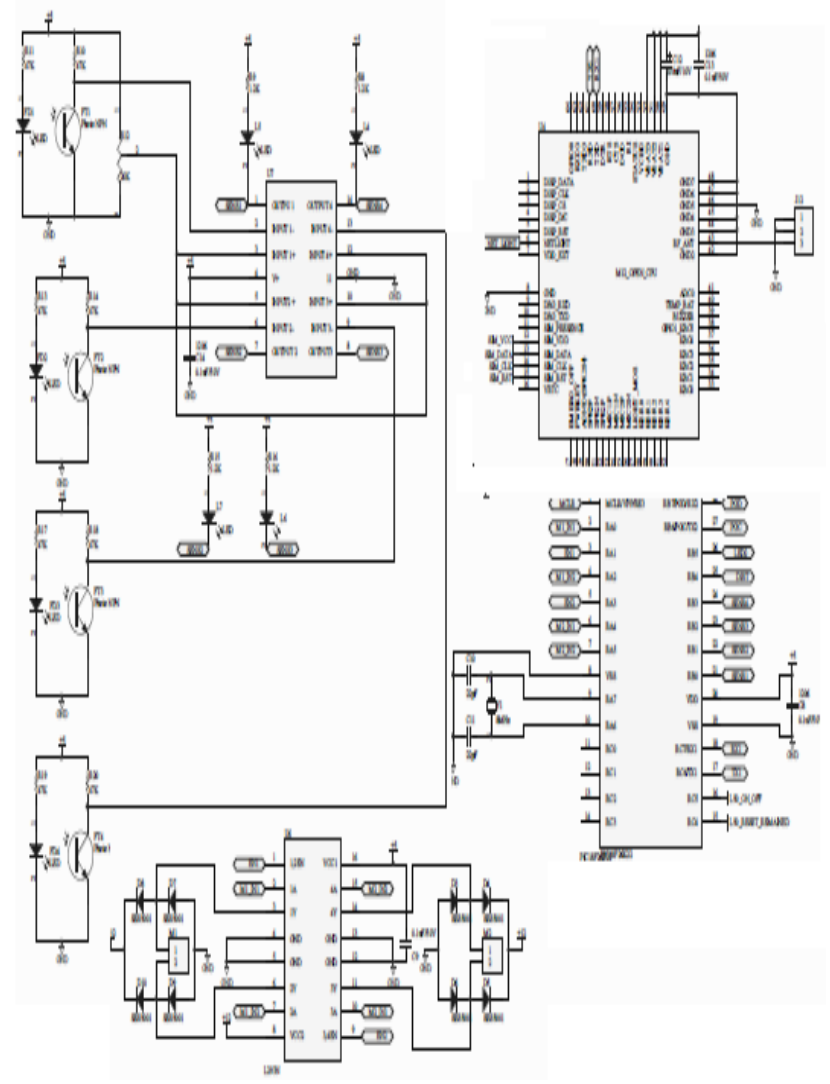

Fig-.9. Interfacing Diagram [12]

Quectel L50 Read only memory (ROM) based GPS used in this system. L50 has fast tracked \& acquisition features. The output of GPS L50 has Recommended Minimum Specification, Global positioning system, Global Navigation Satellite System, Dilution of Precision (GNSS DOP) and Active satellite \& GNSS Satellite in view Messages body format. In addition the GPS Module is designed with typical $1.8 \mathrm{v}$ power supply, power consumption in Acquisition, Tracking, Hibernate modes are 45Ma@-130dbm, 35Ma@$130 \mathrm{dbm}$ and $20 \mu \mathrm{A}$ respectively. Receiver type L1, 1575.42 $\mathrm{MHz}$ C/A Code.

\subsection{Emergency Alert}

In case harsh braking is detected microcontroller runs set of AT commands to send GPS location over SMS to predefined numbers. Microcontroller continuously receiving current valid location, i.e. Latitude and Longitude from GPS and monitor variation in speed in case of sudden variation it will announce as accident porn situation and text message sent to end user. [9] 


\subsection{Motor Driver}

Motor driver L293 will drive two separate DC motor independently. M1_IN1 and M1_IN2 are DC motor1 input and M2_IN1 and M2_IN2 are DC motor2 input all are connected to Microcontroller digital output. Firmware logic will make this output low or high to drive the respective DC motor. When left side IR sensor sense black and right IR sensor sense white, then firmware drive only right side DC motor2, if left side IR sensor sense white and right IR sensor sense black then firmware drive only left side DC motor1. When both left and right IR sensor detects black both motor1 and motor 2 will drive simultaneously, while both left and right IR sensor detects white initially only motor 2 will drive for 3 seconds, so it will scan for black area if no detection found firmware drives both motor1 and motor 2 simultaneously and poll for if any black portion is detected.

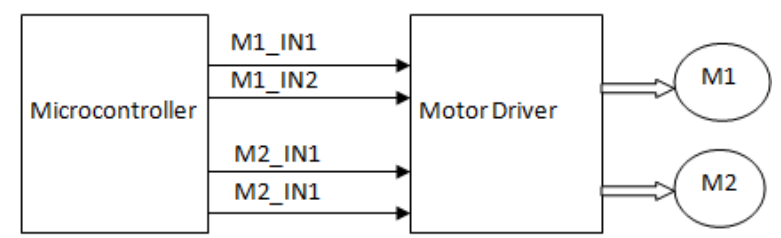

Fig.-10. Motor Driver L293L

\subsection{Software}

All components are built in Microchip MPLAB IDE v8.86, we have written embedded $\mathrm{C}$ codes for accident detection and avoidance. For that we have used Microcontroller General Purpose Input/output (GPIO) port, Uart1, Uart2, timer0 etc. The Code uses the Peripheral support library available with MCC18 Compiler. The Workspace can be easily reconfigured for PIC18F device. Following are the General Guidelines for workspace:

- MPLABIDE >>Configure>>Select Device

- To configure the device settings, either configure the necessary settings from the menu option:MPLABIDE >>Configure >>"Configuration bits"

- Rebuild the MPLAB project using the menu option: MPLAB IDE >>Project $>>$ Build All

- Download the hex file into the device and run

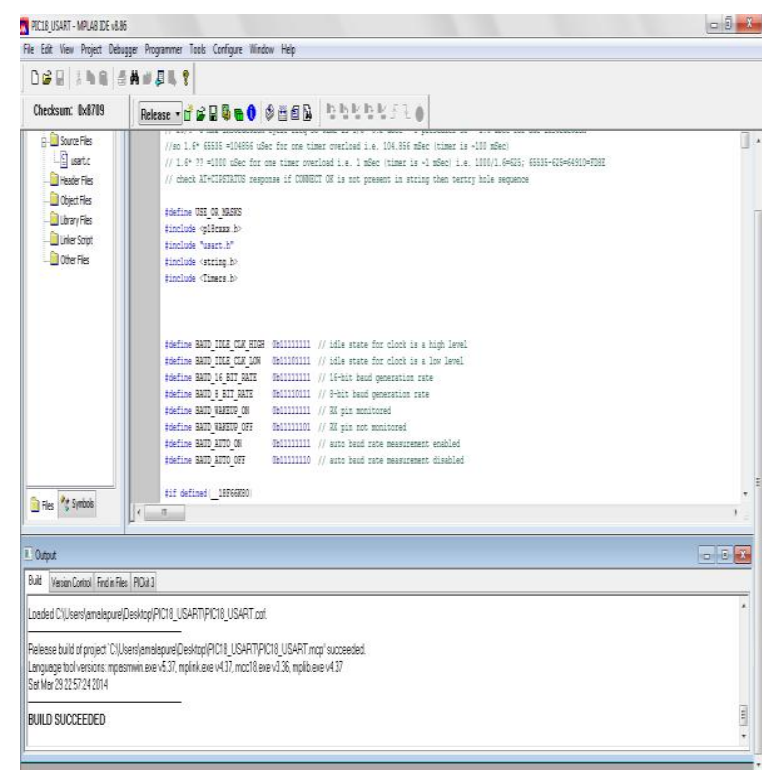

Fig-13. Software Built Successful [12]

\section{FLOW DIAGRAM}

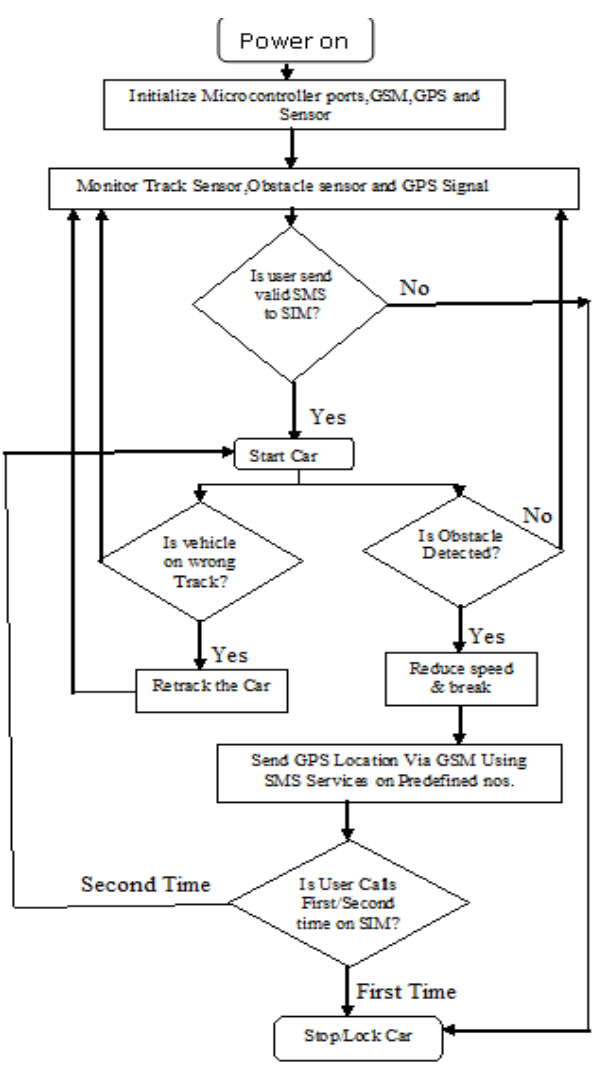

Fig-11.Flow chart of obstacle, track detection \& alert system [12] 
From figure 11, initialize all input and output a port of the Microcontroller and power is supplied to GPS, GSM and sensor modules. If a user sends valid SMS on SIM then only vehicle will start. Otherwise, it it on stable state. [5]Vehicle performs both the functions as obstacle detection as well as track detection ,IR Sensors are placed at the bottom of the car and are used to detect the road track.[8] Due to some unexpected situation, if the car left the track, then system shall automatically break the car and retract the car on proper location.

A Microcontroller is programmed for handling system operation flow it includes digital input, digital output, timers and serial data communication. Microcontroller programming handles GSM and GPS module on its two UARTs respectively, and comparator, motor driver and ultrasonic sensor on digital input /output interface.An Ultrasonic sensor of up to $3 \mathrm{~m}$ ranges is used to detect an obstacle in the front side. If an obstacle is detected then, system shall automatically break and reduce the car speed at the same time an alert SMS with longitude and latitude shall be sent to a predefined Mobile number. If a user calls first time on SIM then the vehicle will stop and in the second call on SIM the car will start automatically.

\section{SPEED MEASUREMENT}

Table -1: SPEED Measurement Look UP TABLE [1] [12]

\begin{tabular}{|c|c|c|}
\hline $\begin{array}{l}\text { Initial } \\
\text { Speed }\end{array}$ & $\begin{array}{l}\text { Max.Speed } \\
\text { after } 1 \mathrm{Sec}\end{array}$ & Result \\
\hline $3 \mathrm{kph}$ & Below $2 \mathrm{Kph}$ & $\begin{array}{l}\text { The Message "\$GPRMC,Time } \\
\text { (hhmmss) 101948.000,latitude, } 184 \\
\text { 5.5306,longitude,07345.3899,spe } \\
\text { ed,1.43, Angle } \\
\text { 188.38 Date180314, A*6A" is } \\
\text { displayed successfully on mobile } \\
\text { screen }\end{array}$ \\
\hline $2 \mathrm{kph}$ & Below 1kph & $\begin{array}{l}\text { The Message "\$GPRMC, Time } \\
\text { (hhmmss)104610.000, latitude, } \\
\text { 1836.0735, longitude, } \\
07348.9496 \text {, speed, 0.50, Angle } \\
0.00, \text { Date180314,,A*61" is } \\
\text { displayed successfully on mobile } \\
\text { screen }\end{array}$ \\
\hline $1 \mathrm{kph}$ & $1 \mathrm{kph}$ & Ideal Situation \\
\hline
\end{tabular}

\section{ADVANTAGES}

1. Security and remote monitoring of vehicles,

2. The road track detection is easy and Low Cost of implementation
3. Theft is unable to hack vehicle Vehicle after 2 minutes automatically send current location to all predefined numbers.

4. The Processor is independent of the compiler

5. Vehicle search alternate path in case of accident in front side

6. The Owner can stop the car at any speed.

\section{LIMITATIONS}

- One of the major disadvantages is that, this system is used only for front barrier detection.

- Neat \& proper arrangement of track is required.

- The system needs GSM networking for SMS service.

- Systems need GPS Signal for acquiring location from the satellite.

\section{APPLICATIONS}

- Security and remote monitoring of vehicles, especially during military operations.

- Used in automotive and transport vehicles- from lighter vehicles like cars, to heavier Automotives like ships and airplanes.

- Track Follower

\section{EXPERIMENTAL SETUP AND RESULT}

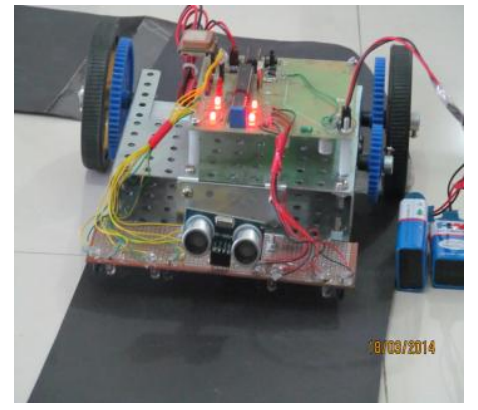

Fig.12 an Obstacle and Track Detection Alert System setup
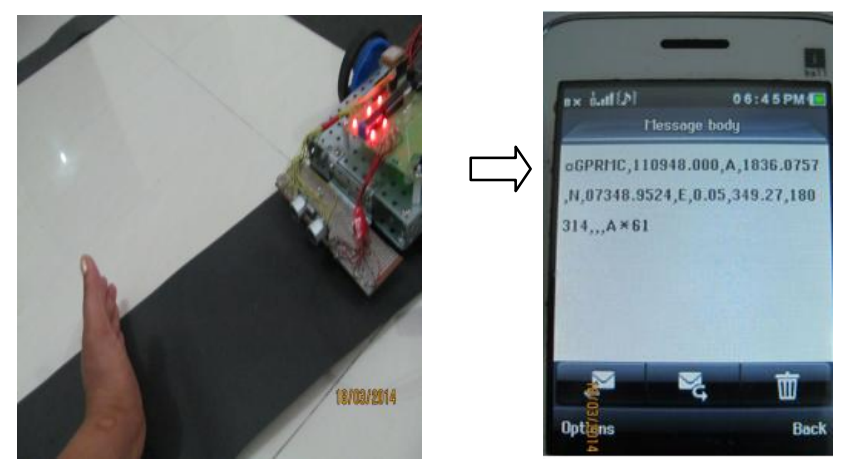

Fig-12.Obstacle detection message [12] 


\section{CONCLUSIONS AND FUTURE WORK}

If the authorized person sends Message on SIM, then only the car starts otherwise lock the car.That means theft unable to hack the car.[11]Any obstacle detects the sensor senses the situation \& car should break slowly \& stop automatically.If the car is on the wrong track then breaking car slowly \& follows the right track automatically.An alert SMS with predefined message body sent to the concert person. Also we have tested this system for different types of obstacles such as other vehicle, human, small stones or any barriers. Track sensor is like a line follower robot.As a part of testing, we have considered all possible scenarios to track. Track arrangement for a vehicle may a straight line track,winding track,number eight like track, triangular track, etc.

The System may be implemented in back side as well as rear sideThis can be interfaced with a vehicle air bag system. That prevents vehicle occupants from a striking interior object such as the steering wheel or window.when the sensors Detect the accident, the air bags get opened.[10] Also, this accumulates camera to capture images inside the vehicle, the camera shall come into action as soon as accident detection triggered and captured images send to predefine email ID's as an attachment with location detail as text in the mail using GPRS service.

\section{ACKNOWLEDGEMENTS}

The authors wish to thank Prof.S.S.Khot Principal, for providing necessary facilities towards carrying out the work. With deep sense of gratitude I express sincere thanks to the Head of the Department Prof.M.S.Biradar and Project Guide Prof.J.R.Panchal who had a faith in this project, believed in my technical work, whispered the words of encouragement \& made helpful suggestions from time to time.Also,thanks to staff member of the Electronics \& Telecommunication Department for their full cooperation \& help.

\section{REFERENCES}

[1] Md.Syedul Amin, Jubayer Jalil and M.B.Reaz. "Accident Detection and Reporting System using GPS, GPRS and GSM Technology" IEEE/OSA/IAPR International Conference on Informatics, Electronics \& vision 2012.978-1-4673-11540/12/31.0@2012IEEE/ICIEV 2012.

[2] Sri Krishna Chaitanya Varma,Poornesh,Tarun Varmaand Harsha "Automatic Vehicle Accident Detection And Messaging System Using GPS and GSM Modems"International Journal of Scientific \& Engineering Research, Volume 4, Issue 8, August2013.1937 ISSN 2229-5518

[3] V.Ramya, B. Palaniappan, K. Karthick “ Embedded Controller for vehicle In-Front Obstacle Detection and Cabin Safety Alert System"IJCSIT Vol 4,No 2,April 2012
[4] Md.Khaled Hossain, Sayed Samial Haq "Detection of Car Pre-crash with Human,Avoidance System \& Localizing through GSM" International Journal of Scientific \& Engineering Research Publication, Volume 3,Issue7,July 2013.

[5] R.Ramani,S.Valarmathy,Dr.N.SuthanthiraVanitha,S.Se lvaraju,Thiruppathi, R.Thangam"Vehicle Tracking and Locking System Based on GSM \& GPS" I.J.ISA,Volume 9,10th Sept 2013

[6] Srudeep Somnaath T K "Autonomous Remote Control Car with Lane Detection and collision avoidance System"IJITEE,Volume 3, Issue 5,October 2013.

[7] S.Saravanan, T.Kavitha "Vehicle Navigation and obstacle Detection System Using RFID and GSM"JATIT, Volume 38, Issue 2, April 2012.

[8] Sawant Supriya C, Dr. Bombale U. L., Patil T.B “An Intelligent Vehicle Control and Monitoring Using Arm"IJEIT, Volume 2, Issue 4, October 2012.

[9] D. Narendar Singh and Ravi teja ch.v. "Vehicle Speed Limit Alerting and Crash Detection System at Various Zones "International Journal of Latest Trends in Engineering and Technology (IJLTET) IJLTET.Vol. 2 Issue 1 January 2013. ISSN: 2278-621X

[10] S.P. Bhumkar, V.V.Deotare and R.V.Babar "Intelligent Car System for Accident Prevention Using ARM7"IJETAE, ISSN 2250-2459, Volume 2, Issue 4, April 2012.

[11] Narasimhulu M, Naresh Kumar Reddy.B.Subrahmanya Sharma.G." Designing of a smart car using arm7" IJAET, ISSN: 2231-1963, Vol. 3, Issue 2, pp. 185-191

[12] Sangita N.Gujar,"Smart car system using Sensor,GPS and GSM"ICST-2K14,Volume 2 